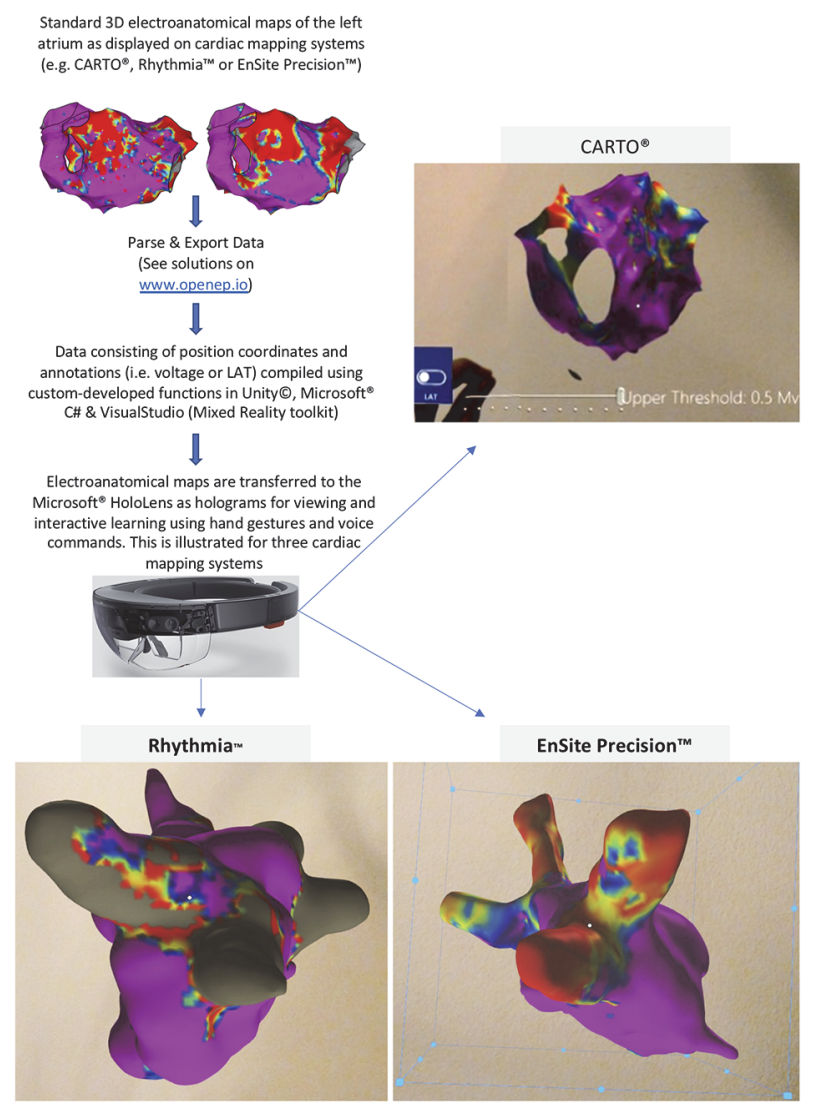

Abstract 107 Figure 1

the left atria had been generated using different mapping systems (CARTO®, Rhythmia ${ }^{\mathrm{TM}}$ and EnSite Precision ${ }^{\mathrm{TM}}$ ) were utilised. These EAMs consisting of 3D coordinates and annotations (e.g. voltage \& activation times) were exported from the mapping system. EAMs were then compiled and transferred to the HoloLens using custom-developed functions on Unity $\left(\right.$ ), Microsoft ${ }^{\circledR}$ C\# and VisualStudio. Subsequently, feedback was obtained from 3 independent electrophysiologists on this technology.

Results We successfully exported the EAMs generated on CARTO ${ }^{\circledR}$, Rhythmia $^{\mathrm{TM}}$ and EnSite Precision ${ }^{\mathrm{TM}}$ mapping systems as holograms on to the HoloLens (Figure). Positive feedback included themes such as 1) the ability to use hand gestures and voice commands to interact with EAMs independent of another user unlike traditional cardiac mapping systems 2) offering an interactive 3D holographic experience whilst preserving the operators' physical interaction in the cardiac catheter lab 3) the capacity to better appreciate 3D geometry of EAMs in comparison to $2 \mathrm{D}$ monitors. The challenge of wearing a headset during long procedures was perceived as a disadvantage.

Conclusion This technology, which can be used with any mapping system, is currently optimised for offline display. Our software will be made available as an opensource teaching and simulation tool (at www.Openep.io). Users will be able to explore EAMs for research, planning complex cases and immersive learning. The future directions will include extending this toolkit for real-time cardiac mapping with catheter localisation, and could potentially be translated to other cardiac imaging modalities.

Conflict of Interest None

\section{SYSTEMIC REVIEW OF ALLERGIES AND TREATMENT TO COMPONENTS OF CARDIAC IMPLANTABLE ELECTRONIC DEVICES}

Tharindra Dissanayake, Parin Shah, Andrei Margulescu, Dewi Thomas, James Barry. Swansea Bay University Health Board, Swansea, UK

\subsection{6/heartjnl-2021-BCS.107}

Background Components of Cardiac Implantable Electronic Devices (CIEDs) consist of polymers and metallic alloys. Although they are rare, there are documented cases of allergic reactions to components of CIEDs. These are commonly misdiagnosed as device pocket infections leading to wasted resources and potentially serious consequences for the patient. Methods We conducted a systematic literature review to identify reported cases of allergic reactions to components of CIEDs and their treatment/management. The words 'pacemaker', 'cardiac implantable electronic device', 'cardiac rhythm device', 'implantable cardiac defibrillator' or ' cardiac resynchronization therapy' in combination with 'replacement' or 'treatment' were used as search criteria.

Results We identified a total of 32 case reports of Allergic reactions to components of CIEDs. Of these 25 were PPMs (Permanent Pace Makers) and 7 were ICDs (Implantable Cardioverter Defibrillator) (1 CRTD - Cardiac Resynchronization Therapy Device). 18 reported hypersensitivity reactions were to alloys or metals and 13 , due to polymers. In 1 case, patch testing showed positivity to multiple allergens but a single, causative material was not identified. The onset of symptoms as a result of these reactions showed wide variation, ranging from 1 day to 10 years post CIED implant. The most common metal allergen was Titanium and the most common polymer was Epoxy Resin. Patient's history, clinical examination and patch testing were used to diagnose allergic reactions to CIED components. Management of the allergic reactions varied depending on the severity. Topical steroids were used for limited cutaneous reactions, while in more deep seated reactions, a complete system extraction with re-implantation of a Gold coated CIED was performed.

Conclusion Allergic reactions to CIEDs are rare but important complications. It is important to identify this pathology early in order to manage patients optimally to minimize harm.

Conflict of Interest None

\section{IMPACT OF COVID-19 PANDEMIC ON IN-PATIENT MORTALITY OF PATIENTS WITH IMPLANTABLE CARDIAC DEVICES}

Ronald Manorekang, Vikrant Nayar. The Mid Yorkshire Hospitals NHS Trust, Wakefield, UK

\subsection{6/heartjnl-2021-BCS.108}

Background Patients with cardiovascular disease have a higher risk of death from COVID-19 infection. Most patients with implantable cardiac devices (pacemakers and defibrillators) have significant cardiovascular co-morbidities, which would suggest a higher mortality in device patients during the pandemic.AimTo review inpatient mortality of patients with cardiac devices in our hospital before and after the outbreak of the COVID-19 pandemic.MethodA retrospective analysis of all in-hospital deaths over 12 months, between 1st November 2019 and 31st October 2020, at a district general hospital in the UK. All patients with implantable cardiac devices were 
Inpatient Deaths of Patient with Cardiac Devices

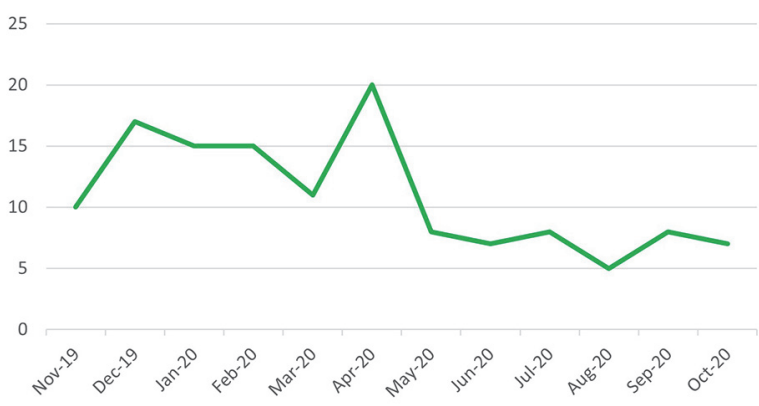

Abstract 109 Figure 1

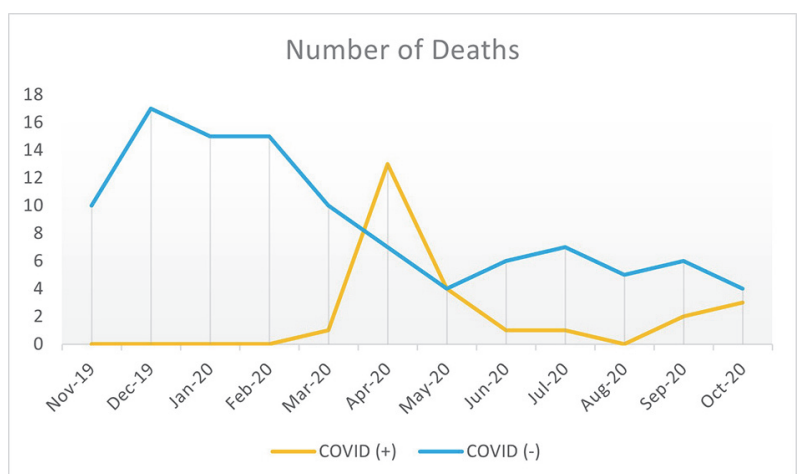

Abstract 109 Figure 2

identified and their COVID-19 infection status determined from the death certificate.

Results 131 in-patients (76\% male) with implantable cardiac devices (64\% pacemakers, 23\% biventricular pacemakers, and $13 \%$ defibrillators) died during the study period. Mean age of death was 84 years (SD \pm 8 years). Most deaths (91\%) occurred on the medical ward (including level 2 respiratory care unit). There were more deaths from March 2020, peaking in April 2020, before gradually declining in May 2020, correlating with the first wave of the COVID-19 pandemic in the UK (figure 1). The majority of patients who died in April 2020 were COVID positive. From early September 2020 onwards, the number of COVID positive deaths started to increase again, correlating with the second wave. Following the declaration of the pandemic, deaths occurred mostly amongst COVID negative patients (figure 2).

Conclusion Inpatient mortality of patients with cardiac device were highest in the elderly population which predominantly occurred during the first wave of COVID-19 pandemic.

Conflict of Interest none

\section{SYSTEMATIC REVIEW OF SAFETY AND EFFICACY OF RENAL SYMPATHETIC DENERVATION FOR THE MANAGEMENT OF CARDIAC ARRHYTHMIAS}

Nakulan Nantha Kumar. University of Bristol, Bristol, UK

10.1136/heartjnl-2021-BCS.109

Background Renal sympathetic denervation (RSD) has an emerging role in treating cardiac arrythmias, especially Atrial
Fibrillation (AF), as reported by several studies and due to its role in reducing blood pressure and speculation on its effects on the autonomic nervous system. In the wake of the controversy surrounding the SMYPLICITY HTN-3 trial and data from subsequent trials, this review aims to perform an updated and more comprehensive review of the impact of RSD on atrial and ventricular arrhythmias. Method: A systematic search included the Medline, Scopus and Embase databases using the terms 'Renal Denervation' AND 'Arrhythmias or Atrial or Ventricular', limited to Human and English language studies within the last 10 years. This search yielded, 19 relevant studies $(n=6$ randomised controlled trials, $n=13$ nonrandomised cohort studies) which comprised 783 patients.

Results Upon analysis, the data shows RSD is a safe procedure and is not associated with increases in complications or mortality post procedure. Importantly, there is no evidence RSD is associated with a deterioration in renal function, even in patients with chronic kidney disease. RSD with or without adjunctive Pulmonary Vein Isolation (PVI) was associated with improvements in freedom from AF, premature atrial complexes (PACs), Ventricular arrythmias and other Echocardiographic parameters. Significant reductions in both ambulatory and office blood pressure were also observed in the majority of studies.

Conclusion The findings of this review support the findings of so called 'second generation' RSD studies which report that RSD is safe and is associated with reductions in short term blood pressure, AF burden and key Echocardiological indices like LVEF. However, there is a paucity of evidence regarding the impact of RSD on other less prominent arrythmia subtypes. Hence, large standardized multi-centre RCTs investigating the role of RSD are necessary to comprehensively assess the efficacy of the procedure in treating all arrythmias. (300 words)

Conflict of Interest N/A

\section{A TERTIARY CENTRE EXPERIENCE COMPARING NEW TEMPERATURE-CONTROLLED HIGH-POWER SHORT- DURATION ABLATION TECHNOLOGY WITH STANDARD RADIOFREQUENCY AND CRYO-ENERGY ABLATION}

${ }^{1}$ Akash Mavilakandy, ${ }^{1}$ Bharat Sidhu, ${ }^{2}$ Sharon Man, ${ }^{1}$ Ibrahim Antoun, ${ }^{1}$ Zakarriya Vali, ${ }^{1}$ Tiago Paggi De Almeida, ${ }^{1}$ Xin Li, ${ }^{2}$ Merzaka Lazdam, ${ }^{2}$ Mokhtar Ibrahim, ${ }^{2}$ ShuiHao Chin, ${ }^{2}$ Alastair Sandilands, ${ }^{2}$ Peter Stafford, ${ }^{2}$ Riaz Somani, ${ }^{1}$ Andre Ng. ${ }^{1}$ University of Leicester, Leicester, UK; ${ }^{2}$ University Hospitals of Leicester

\subsection{6/heartjnl-2021-BCS.110}

Introduction Pulmonary vein isolation (PVI) is an established treatment strategy for atrial fibrillation (AF). Radiofrequency (RF) ablation technology has evolved over the last decade with the very high-power short-duration (vHPSD) temperature-controlled ablation approach emerging as the latest development. The aim of this study was to determine the procedural efficacy, metrics and safety of vHPSD ablation with conventional power-controlled RF (PCRF) ablation and cryoenergy ablation (CRYO).

Methods A prospective single tertiary centre analysis was performed on patients undergoing first time PVI ablation from 2019 to 2020. The population was divided in to 4 treatment arms; QMode Plus (vHPSD), Qmode (Qmode), PCRF using THERMOCOOL SMARTTOUCH @ SF Catheter (STSF) and CRYO. Demographics, clinical and procedural metrics such as PVI duration, ablation time and sedation requirement were 